
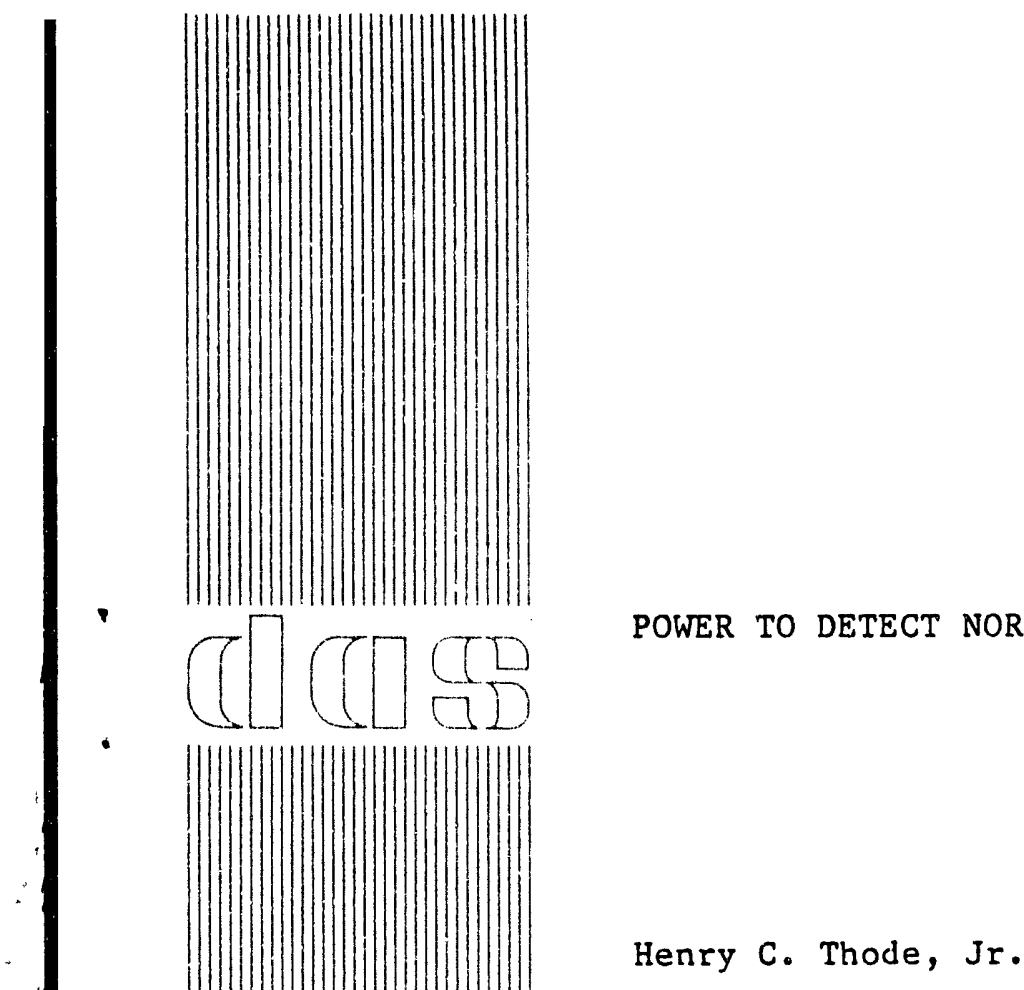

July 1992

Henry C. Thode, Jr.
BNL -47929

INFORMAL REPORT

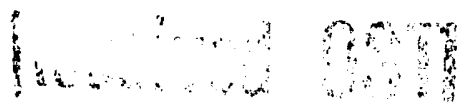

NoV 201992

POWER TO DETECT NORMAL MIXTURES: SIMULATION RESULTS

BROOKHAVEN NATIONAL LABORATORY UPTON, LONG ISLAND. NEW YORK 11973 


\section{DISCLATMER}

This report was prepared as an account of work sponsored by an agency of the United States Government. Neither the United States Government nor any agency thereof, nor any of their employees, nor any of their contractors, subcontractors, or their employees makes any warranty, express or implied, or assumes any legal liability or responsibility for the accuracy, completeness, or usefulness of any information, apparatus, product or process disclosed, or represents that its use would not infringe privately owned rights. Reference herein to any specific cownercial product, process, or service by trade name, trademark, wanufacturer, or otherwise, does not necessarily constitute or imply its endorsement, recomendation, or favoring by the United States Government or any agency thereof. The views and opinions of authors expressed herein do not necessarily state or reflect those of the Onited States Government or any agency, contractor, or subcontractor thereof. 
BNL- -47929

DE93 003143

POWER TO DETECT NORMAL MIXTURES: SIMULATION RESULTS

Henry C. Thode, Jr.

July 22, 1992

Department of Applied Science

Brookhaven National Laboratory

Upton, Long Island, New York 11973

MASTER

This research was performed under the auspices of the United States

Department of Energy Under Contract No. DE-AC02-76CH00016 


\title{
Power to Detect Normal Mixtures: Simulation Results
}

\author{
Henry C. Thode, Jr.
}

\section{Summary}

Twenty tests for normality were compared for the purpose of detecting mixtures of two normal components with unequal means but equal variance. The purpose of this study was to determine the power of tests specifically designed to detect mixtures, i.e., the likelihood ratio and Engelman-Hartigan tests, relative to other tests for normality. We considered the entire range of mixing proportions $\pi, 0<\pi<1$. For mixtures that are nearly symmetric $(0.35<\pi<0.65)$ the Engelman-Hartigan test was the most powerful. When $\pi>0.85$ or $\pi<0.15, \sqrt{b_{1}}$ was among the best tests. For intermediate mixing proportions, the likelihood ratio test was best. For situations in which the preferred test had power $50 \%$ or more, the power of the likelihood ratio test was also above $50 \%$ and within 15 percentage points of the preferred test.

\section{Introduction}

In this paper we examined tests with respect to their power to detect whether observed data are a random sample from a normal distribution or from a mixture of two normal distributions with equal standard deviations but different means. The probability density function of this mixture is given by

$$
f(x)=\pi \frac{1}{\sigma} \phi\left(\frac{x-\mu_{1}}{\sigma}\right)+(1-\pi) \frac{1}{\sigma} \phi\left(\frac{x-\mu_{2}}{\sigma}\right)
$$

where $\phi(z)$ denotes the standard normal density with mean 0 and variance $1, \mu_{1}$ and $\mu_{2}$ are the respective means of the components with $\mu_{1}<\mu_{2}, \sigma$ is the common standard deviation of the components, and $\pi$ is the mixing proportion.

Researchers in genetics have used a wide variety of other procedures, but there have been few comparisons of the methods used to establish empirically the existence of these mixtures. Elston et al. (1974) used tests of skewness $\left(\sqrt{b_{1}}\right)$ and kurtosis $\left(b_{2}\right)$ as their first step in demonstrating that a single normal distribution did not fit the data. Lee (1975) found that $b_{2}$ was more powerful than the Engelman-Hartigan (EH) test when $\pi$ 
was near 0 or 1 , but when $\pi$ was close to $0.50 \mathrm{EH}$ was more powerful. Everitt and Hand (1981), Titterington, Smith and Makov (1985) and McLachlan and Basford (1988) contain extensive bibliographies on the subject of finite mixture distributions (including normal mixtures) and a wide range of applications from a variety of disciplines.

We have focused our attention in this paper on tests formally defined in the statistical literature. Many investigators have used visual inspections of the histograms, but the subjectivity and complexity of inspection as practiced make this a difficult procedure to study by simulation. In addition, we have not included the procedure of Maclean et al. (1976), wherein a scaled version of the Box and Cox (1964) transformation is used to remove skewness and the distribution of the transformed data is tested for fit to a mixture of two normal components.

\section{Methods}

\subsection{Tests Used in Study}

Twenty tests for normality or goodness of fit were used in this power comparison study (Table 1). These were classified as either (a) tests for mixtures; (b) moment-type tests; (c) regression and correlation tests; (d) empirical distribution function (EDF) tests; (e) outlier tests; or (f) other types of tests.

\subsubsection{Tests for Mixtures}

The likelihood ratio test (LRT) is a general statistical procedure that can be applied to generate a test of this hypothesis. The likelihood ratio test is the maximum value obtained for the logarithm of the ratio of the likelihood of the normal mixture distribution to that of the single normal distribution, i.e.,

$$
G^{2}=\log \left[L_{1} / L_{0}\right]=\log \left[L_{1}\right]-\log \left[L_{0}\right]
$$

where

$$
\begin{gathered}
L_{1}=\prod_{i=1}^{n} f(x), \\
L_{0}=\prod_{i=1}^{n} \phi\left(\frac{x-\bar{x}}{s}\right),
\end{gathered}
$$

$\phi(y)$ is the standard normal distribution and $\bar{x}$ and $s$ are the estimated mean and standard deviation. Since there is no closed form solution to the maximum of $G^{2}$, iterative methods are necessary to calculate the test. Everitt (1981) and McLachlan (1987) used simulation to find the power of the LRT for mixtures of two components and Mendell, Thode, and Finch (1991) studied the power and sample size requirements for the LRT.

However, comparisons of the LRT with other tests for normality have not been performed.

The Engelman-Hartigan test ( $\mathrm{EH})$ is a test based on a result from the methodology of clustering techniques (Engelman and Hartigan, 1969). For this test, the data are divided 
into two clusters and the sum of squares within clusters $\left(S S_{W}\right)$ and between clusters $\left(S S_{B}\right)$ are obtained. The test is calculated as

$$
E H=\max \left[S S_{B} / S S_{W}\right]
$$

where the maximum is taken over all possible divisions of the data into two groups. The maximum occurs for one of the $n-1$ partitions of the sorted data.

\subsubsection{Moment and Absolute Moment Tests}

Standardized sample moments obtained from a sample are

$$
m(k)=\frac{\sum\left(x_{i}-\bar{x}\right)^{k}}{n s^{k}}
$$

and are often used as tests of normality, with skewness $\left(m(3)=\sqrt{b_{1}}\right)$ and kurtosis $(m(4)=$ $b_{2}$ ) being the most widely known and used. Skewness is a powerful test of normality over a wide range of skewed distributions, and also is the locally best invariant one-sided test for outliers when all are in the same direction (Ferguson, 1961), so that skewness is expected to be a good test for detecting asymmetric normal mixtures. Kurtosis is powerful over a wide range of symmetric alternatives. Box (1953) indicated that higher order $(>2)$ even moment tests such as kurtosis should be good for detecting short-tailed symmetric alternatives such as symmetric $(\pi=0.50)$ mixtures (Figure 1$)$.

A combined test of skewness and kurtosis (D'Agostino and Pearson, 1973; 1974),

$$
K^{2}=X^{2}\left(\sqrt{b_{1}}\right)+X^{2}\left(b_{2}\right)
$$

was included in this study where $X\left(\sqrt{b_{1}}\right)$ and $X\left(b_{2}\right)$ are transformations to standard normality of the skewness and kurtosis tests, respectively. The transformations used here were those of D'Agostino (1970) for skewness and Anscombe and Glynn (1983) for kurtosis.

Two higher order moment tests, $m(5)$ and $m(6)$ were also used in this study. As an odd moment test, $m(5)$ should have properties similar to $\sqrt{b_{1}}$. As an even moment test, $m(6)$ should be similar to kurtosis.

Even moment tests are also a subset of the class of absolute moment tests

$$
a(k)=\frac{\sum\left|x_{i}-\bar{x}\right|^{k}}{n s^{k}} .
$$

Geary's test $a(1)$ (often denoted $g$ ) is the most well known of these tests (Geary, 1935); Box (1953) indicated that absolute moment tests with $0<k<2$ might be better at detecting long-tailed symmetric alternatives to the normal; in particular, $a(1)$ is asymptotically equivalent to the likelihood ratio test for detecting a double exponential alternative to the normal distribution. The tests $a(3)$ and $a(5)$, which should have properties similar to kurtosis, were also included in this study. 


\subsubsection{Regression and Correlation Tests}

The Wilk-Shapiro test $W$ (Shapiro and Wilk, 1965) is one of the most well known tests for normality. $U_{p}$ to a constant, $W$ is the ratio of the estimated squared slope of a normal probability plot to the usual estimated variance; under normality, the slope is an estimate of the standard deviation. Although originally developed as an omnibus test, many power studies show that $W$ is more powerful against skewed alternatives than symmetric alternatives relative to other tests. Due to difficulties in calculating $W$ for large samples, an approximate $W$ test, the Shapiro-Francia $W^{\prime}$ (Shapiro and Francia, 1972), was used in this study for samples of size 100.

Filliben's probability plot correlation $\left(r_{F}\right)$ is the simple Pearson correlation of the probability plot where the plotting positions are the median of the normal order statistics (Filliben, 1975). This test is asymptotically equivalent to $W$, and should have similar power properties.

\subsubsection{Outlier Tests}

Outlier tests were included because Ferguson (1961) used a normal mixture model to derive outlier tests and Aitkin and Wilson (1980) used a normal mixture model to describe a sample with outliers. Two outlier tests were included in this study: Grubbs' test $T$ (Grubbs, $1950 ; 1969)$ and Dixon's $r_{10}^{(2)}$ test (Dixon $1950 ; 1951$ ). Both of these tests are designed to detect a single outlier in an unspecified direction. Intuitively, these tests should be most powerful for detecting mixtures where $\pi$ is small relative to $n$. In addition, $T$ is similar to the range test $u$ (see below), so that it is potentially useful as a test for detecting short-tailed symmetric distributions (Thode, 1985).

\subsubsection{EDF Tests}

EDF tests are based on comparisons of the empirical and hypothesized distribution function of the random variable. Although originally developed as tests of the simple hypothesis, these tests have been modified so that they can be used as tests of fit when the parameter values of the data distribution are unknown.

The Kolmogorov-Smirnov $\left(D_{K S}\right)$ test (Lilliefors, 1967) is perhaps the most well known of the EDF tests, and is a distance test; the Cramer-von Mises $\left(W^{2}\right)$ test (Anderson and Darling, 1952) and the Anderson-Darling $\left(A^{2}\right)$ test (Anderson and Darling, 1954) are quadratic tests, based on functions of the squared differences. The Anderson-Darling test weights differences in the tails of the distributions more heavily than does $W^{2}$. The Anderson-Darling test in particular has been shown to be generally competitive with the Wilk-Shapiro test in testing for normality (Stephens, 1974).

\subsubsection{Other Tests for Normality}

The range test $u$ (David, Hartley, and Pearson, 1954) is the likelihood ratio test for detecting a uniform alternative to the normal distribution, and therefore one might suspect it would also be useful at detecting other short-tailed alternatives, e.g., the symmetric mixtures studied here.

D'Agostino (1971) proposed $D_{a}$ as an alternative omnibus test to $W$ for large samples due to the computational difficulties with $W$; similar to $W$, it is also the ratio of two 
estimates of $\sigma^{2}$. Unlike $W$, though, $D_{a}$ seems to be a better test at detecting long-tailed symmetric alternatives, especially when used as a directional (single-tailed) test.

The $\chi^{2}$ test is probably the most well known and most commonly used goodness of fit test, since it is adaptable to any null distribution. However, as a test for normality it is rarely competitive with other tests in terms of power.

\subsection{Simulation Methods}

Power was determined for three sample sizes $(n=25,50,100)$, eight mixing proportions $(\pi=0.50,0.60,0.65,0.70,0.80,0.85,0.90,0.95)$, and selected values of $D=\frac{\left(\mu_{2}-\mu_{1}\right)}{\sigma}$ for each sample size. Due to the symmetry of the mixture with respect to $\pi$ (i.e., results for $\pi=\pi_{0}$ are the same as for $\pi=1-\pi_{0}$ ), without loss of generality we restricted our attention to values of $\pi=0.50$ or greater. The number of replications used for each combination $(\pi, D, n)$ was 5000 except for situations in which the best test had power close to $100 \%(n=50, D=5 ; n=100, D=4,5)$ where 1000 samples were used.

Samples were obtained by generating two uniform random numbers for each random value from the mixture sample. One random number was transformed into a normal deviate, and the other was used to allocate the deviates to the components of the alternative according to the specified value of $\pi$. Since the tests we considered are location and scale invariant, it was assumed without loss of generality that the component variance was 1 and the mean of the first component was 0 . The random numbers were obtained from a uniform sequence using the congruential generator and random shuffle of the resulting sequence (Andrews et al., 1972).

For the LRT, EH test, $r_{F}$ and $W$ we used single-tailed tests at the .05 level. For the LRT and EH tests, large values indicate departures from normality, so upper tail critical values were used. As correlation tests, $W$ and $r_{F}$ indicate nonnormality when values are low, so lower tail critical values were used. The coefficients for calculating $W$ contained in Shapiro and Wilk (1965) were used for $n=25$ and 50. For $n=100$, the Shapiro-Francia test $W^{\prime}$ was used. For the $\chi^{2}$ test, equiprobable cells were used, with the number of cells for sample sizes 25,50 and 100 equal to 6,9 and 9, respectively. The EDF tests were also used as upper-tail tests, ignoring the possibility of superuniformity (e.g., Stephens, 1986), which was not considered a problem. For the moment tests, absolute moment tests, $u$, the outlier tests and $D_{a}$, two-tailed .05 level tests were used; although these tests were at a disadvantage under two-tailed testing, the simulation was performed under the assumption that the values of $\pi$ and $D$ were not known in advance. Therefore, the population skewness could be positive or negative as $\pi$ is less than or greater than 0.50 (Figure 2), and population kurtosis could be greater than (for $0.20<\pi<0.80$, approximately) or less than (for $\pi<0.20$ or $\pi>.80$ ) the normal value of 3 (Figure 1 ).

For the LRT, the empirical percentage points for the null distribution reported by Thode, Finch, and Mendell (1988) were used. For each of the EDF tests, the sample size adjustment formula and upper-tail .05-level critical value reported by Stephens (1986) were used. Critical values for the $\chi^{2}$ test were obtained from a standard distribution table. $K^{2}$ was assumed to be distributed as $\chi_{2}^{2}$ for all sample sizes. All other critical values were obtained by generating 5001 normal samples of each sample size, applying each test to the 
samples and determining the critical value(s) for each test at the appropriate level.

\section{Results}

The LRT was determined to be the best test to use of the twenty studied here when no information is available on the true value of $\pi$ (Tables $2,3,4$ ). For $\pi<0.65$, the LRT was second to EH, while for $0.65 \leq \pi<0.85$, the LRT was always the most powerful test. For $\pi>0.85$ its power was never more than 12 percentage points less than the best test, and when the powrer of the best test was appreciable the LRT was usually within 5 percentage points.

The EH test was conspicuously the most powerful for mixtures with $\pi=0.50$ and was somewhat more powerful than the LRT up to about $\pi=0.65$. The largest percentage point difference between the EH test and the LRT was for $\pi=0.50, D=3$, and $n=50$, where the power of the EH test was $75.9 \%$ compared to $59.5 \%$ for the LRT. In general, as the sample size or $D$ increased for fixed $\pi$ in this range, the difference between the powers of the two tests became smaller. When $\pi=0.65$, the LRT had about the same power as $\mathrm{EH}$. For larger $\pi$ the power of the EH test decreased. In fact, when $\pi$ was near 1 , the EH test had power less than the level of significance in some situations, i.e., the EH test is biased.

The skewness test was among the most powerful of these twenty tests when $\pi>0.85$. Its closest competitors were $r_{F}$ when $\pi=0.90$, and $m(5)$ and $r_{F}$ when $\pi=0.95$. The sampling situation in which the percentage point difference between $\sqrt{b_{1}}$ and the LRT was largest was $\pi=0.95, D=3$ and $n=100$. The power of $\sqrt{b_{1}}$ was $67.7 \%$ compared to an estimated power of $57.4 \%$ for the LRT. There was also a ten percentage point difference for $\pi=0.90, D=3$, and $n=50: 59.9 \%$ power for $\sqrt{b_{1}}$ compared to $49.6 \%$ estimated power for the LRT. Both $\sqrt{b_{1}}$ and $m(5)$ are biased when the mixture is near symmetric.

Kurtosis was rarely competitive with the most powerful test at any specified value of $\pi$ and was less powerful than the LRT in general. When $\pi=0.50$ or $\pi=0.95, b_{2}$ was relatively more effective because the value of the fourth cumulant is farthest away from 0 for these mixing proportions; it had lowest power for fixed sample size and $D$ when $\pi=0.80$. Geary's test was less powerful than the LRT except when $\pi=0.50$.

All of the absolute moment tests followed the pattern observed for kurtosis with respect to $\pi$. Compared to each other, $a(1)$ was consistently more powerful for $0.50 \leq$ $\pi \leq 0.85$, followed by $a(3)$, kurtosis, $a(5)$ and $m(6)$, indicating that power decreases for higher rnoment tests. No consistent pattern among absolute moment tests was observed for $\pi>0.85$.

The power of $W$ was close to the power of the LRT. When $0.30 \leq \pi \leq 0.70$, the LRT is more powerful than $W$ for smaller sample sizes. The two tests had about the same power when $0.80 \leq \pi \leq 0.90$, and $W$ was slightly more powerful than the LRT when $\pi=0.95$. These findings are consistent with Shapiro, Wilk, and Chen (1968) who found that $W$ had good power for detecting skewed alternative distributions. Filliben's $r_{F}$ was noticeably less powerful than $W$ for $\pi<0.85$, but was among the best tests for the more skewed mixtures.

Among EDF tests, $W^{2}$ and $A^{2}$ were always comparable, although $A^{2}$ tended to have slightly higher power, especially for higher values of $\pi$. $W^{2}$ was never more than 6 per- 
centage points lower than $A^{2}$. $D_{K S}$ always had appreciably less power than either $A^{2}$ or $W^{2} . W^{2}$ and $A^{2}$ had noticeably less power than the LRT and EH for symmetric and near-symmetric distributions but were among the most powerful for moderately skewed mixtures $(0.80 \leq \pi \leq 0.90)$.

Both of the outlier tests examined had negligible power for symmetric and nearsymmetric distributions. $T$ had slightly better power than LRT for samples of size 25 and $\pi=0.95$ and was competitive with LRT for $n=50$ and 100 , although $T$ was never the best test for any value of $D$.

Of $u, D_{a}$ and the $\chi^{2}$ test, none was competitive with the best test for any value of $\pi$. Over all sample sizes, $D_{a}$ had the lowest power of the three tests for $\pi<0.85$; for $n=25$ and near-symmetry, $u$ had higher power than $\chi^{2}$, while for all other situations with $\pi<0.85$ the $\chi^{2}$ test had highest power of the three tests. For the skewed mixtures $D_{a}$ had the highest power of the three tests.

\section{Conclusions}

Two tests were consistently most powerful for some values of the mixing proportion: LRT and EH. The EH test was the most powerful test when $0.35<\pi<0.65$ with the LRT being its closest competitor. Since such mixtures are nearly symmetric and have negative fourth cumulants, the relative effectiveness of absolute moment tests and the lack of effectiveness of $W$ and odd moment tests are expected results. When $\pi=0.50$, the absolute moment tests were the most powerful tests after EH and LRT; as $\pi$ gets larger, the power of these tests relative to $\mathrm{EH}$ decreased, and $A^{2}$ and $W^{2}$ were the most powerful of the general tests for normality.

When $0.65 \leq \pi \leq 0.85$ (or $0.15 \leq \pi \leq 0.35$ ), the LRT was best. For these mixing proportions, the third cumulant of the mixture has a moderate value, and the fourth cumulant is near zero, so that the standard tests of normality (e.g., skewness and kurtosis) would be at a considerable disadvantage compared to the LRT. The Anderson-Darling, Cramer-von Mises and Wilk-Shapiro tests were close competitors of the LRT in this range of mixing proportions.

When $\pi>0.85$ or $\pi<0.15, \sqrt{b_{1}}, K^{2}, r_{F}, W$, and $m(5)$ were about equal in power. A normal mixture of this type is very skewed and has a positive fourth cumulant, so that the effectiveness of skewness, $W$ and related tests would be expected. The relative lack of power of the LRT for small values of $\pi$ was surprising and suggests that the use of the LRT as a test for outliers (as proposed in Aitkin and Wilson, 1980) is not recommended.

For $\pi$ near 0.85 , there was no consistently best test with respect to all $n$ and $D$. This is apparently a transition zone where tests with high power for near-symmetric distributions decrease in power (for fixed $n$ and $D$ ), while tests for skewed distributions begin to peak.

When there is prior information concerning the true value of the mixing proportion, $\mathrm{EH}, \mathrm{LRT}$ and $\sqrt{b_{1}}$ are recommended for detecting mixtures depending upon the hypothesized value of $\pi$. In the absence of prior knowledge, the LRT is recommended since it is always within a respectable distance from the best test at any value of $\pi$.

In this study, two-tailed tests were used for moment tests, $D_{a}, u$ and the outlier tests under the scenario that the direction of skewness of the mixture (i.e., $\pi<0.50, \pi>0.50$ 
or $\pi=0.50$ ) and the population kurtosis of the mixture (i.e., $\beta_{2}<3$ for symmetric, $\beta_{2}>3$ for asymmetric) are not known a priori. If such prior information was available, the use of one-tailed tests could result in a substantial increase in power over that shown in this report. 


\section{References}

Aitkin, M., and Wilson, G.T. (1980). Mixture models, outliers, and the EM algorithm. Technometrics 22, 325-331.

Anderson, T.W., and Darling, D.A. (1952). Asymptotic theory of certain "goodness of fit" criteria based on stochastic processes. Annals of Mathematical Statistics 23, 193-212.

Anderson, T.W., and Darling, D.A. (1954). A test of goodness of fit. Journal of the American Statistical Association 49, 765-769.

Anscombe, F.J., and Glynn, W.J. (1983). Distribution of the kurtosis statistic $b_{2}$ for normal statistics. Biometrika 70, 227-234.

Andrews, D.F., Bickel, P.J., Hampel, F.R., Huber, P.J., Rogers, W.H., and Tukey, J.W. (1972). Robust Estimates of Location: Survey and Advances. Princeton University Press, New Jersey.

Box, G.E.P. (1953). A note on regions for tests of kurtosis. Biometrika 40, 465-468.

Box, G.E.P., and Cox, D.R. (1964). An analysis of transformations (with discussion). Journal of the Roya! Statistical Society B26, 211-252.

D'Agostino, R.B. (1970). Transformation to normality of the null distribution of $g_{1}$. Biometrika 57, 679-681.

D'Agostino, R.B. (1971). An omnibus test for normality for moderate and large sample sizes. Biometrika 58, 341-348.

D'Agostino, R.B. and Pearcon, E.S. (1973). Tests for departure from normality. Empirical results for the distributions of $b_{2}$ and $\sqrt{b_{1}}$. Biometrika 60, 613-622.

D'Agostino, R.B., and Pearson, E.S. (1974). Correction to 'Tests for departure from normality. Empirical results for the distributions of $b_{2}$ and $\sqrt{b_{1}}$, Biometrika 61,647 .

David, H.A., Hartley, H.O., and Pearson, E.S. (1954). The distribution of the ratio, in a single normal sample, of the range to the standard deviation. Biometrika 41, 482-493.

Dixon, W. (1950). Analysis of extreme values. Annals of Mathematical Statistics 21, 488-505.

Dixon, W. (1951). Ratios involving extreme values. Annals of Mathematical Statistics 22, 68-78.

Elston, R.C., Namboodiri, K.K., Nino, H.V., and Pollizer, W.S. (1974). Studies on blood and urine glucose in Seminole Indians: Indications for segregation of a major gene. American Journal of Human Genetics 26, 13-34.

Engelman, L., and Hartigan, J.A. (1969). Percentage points of a test for clusters. Journal of the American Statistical Association 64, 1647-1648.

Everitt, B.S. (1981). A Monte Carlo investigation of the likelihood ratio test for the number of components in a mixture of normal distributions. Multivariate Behavioral Research 16, 171-180. 
Everitt, B.S., and Hand, D.J. (1981). Finite Mixture Distributions. Chapman anci Hall, New York.

Ferguson, T.S. (1961). On the rejection of outliers. Proceedings of the \& th Berkeley Symposium on Mathematical Statistics and Probability, University of California Press, Berkeley CA.

Filliben, J.J. (1975). The probability plot coefficient test for normality. Technometrics 17, 111-117.

Geary, R.C. (1935). The ratio of the mean deviation to the standard deviation as a test of normality. Biometrika 27, 310-332.

Grubbs, F.E. (1950). Sample criteria for testing outlying observations. Annals of Mathematical Sto.tistics 21, 27-58.

Grubbs, F. (1969). Procedures for detecting outlying observations in samples. Technometrics 11, 1-19.

Lee, K.K. (1975). Some Statistical Test for Clusters. University of North Carolina, Institute of Statistics, Mimeo Series No. 1036.

Lilliefors, H.W. (1967). On the Kolmogorov-Smirnov test for normality with mean and variance unknown. Journal of the American Statistical Association 62, 399-402.

MacLean, C. J., Morton, N. E., Elston, R. C., and Yee, S. (1976). Skewness in commingled distributions. Biometrics 32, 695-699.

McLachlan, G.J. (1987). On bootstrapping the likelihood ratio test statistic for the number of components in a normal mixture. Applied Statistics 36, 318-324.

McLachlan, G.J., and Basford, K.E. (1988). Mixture Models: Inference and Applications to Clustering. Marcel Dekker, New York.

Mendell, N.R., Lee, K.L., Reinsmoen, N., Yunis, E., Amos, D. B., and Emme, L. (1977). Statistical methods for evaluating responses in HLA-D typing. Transplantation Proceedings 9, 99-106.

Mendell, N.R., Thode, H.C., and Finch, S.J. (1991). The likelihood ratio test for the two component normal mixture problem: Power and sample size analysis. Biomeirics 47, 1143-1148.

Shapiro, S.S., and Francia, R.S. (1972). Approximate analysis of variance test for normality. Journal of the American Statistical Association 67, 215-216.

Shapiro, S.S, and Wilk, M.B. (1965). An analysis of variance test for normality (complete samples). Biometrika 52, 591-611.

Shapiro, S.S, Wilk, M.B., and Chen, H.J. (1968). A comparative study of various tests for normality. Journal of the American Statistical Association 63, 1343-1372.

Stephens, M.A. (1974). EDF statistics for goodness of fit and some comparisons. Journal of the American Statistical Association 69, 730-737. 
Stephens, M.A. (1986). Tests based on EDF statistics. In D'Agostino and Stephens, eds., Goodness of Fit Techniques, Marcel Dekker, New York.

Thode, Jr., H.C. (1985). Power of absolute moment tests against symmetric non-normal alternatives. Ph.D. dissertation, University Microfilms, Ann Arbor, MI.

Thode, H.C., Finch, S.J., and Mendell, N.R. (1988). Simulated percentage points for the null distribution of the likelihood ratio test for a mixture of two normals. Biometrics 44, 1195-1201.

Titterington, D.M., Smith, A.F.M., and Makov, U.E. (1985). Statistical Analysis of Finite Mixture Distributions. John Wiley and Sons, New York. 
Table 1. Tests Used in Comparison of Power to Detect Normal Mixtures.

Symbol Test

LRT

EH

$\sqrt{b_{1}}$
$b_{2}$
$K^{2}$

$m(5)$

$m(6)$

$a(1)$

$a(3)$

$a(5)$

$W$

$W^{\prime}$

$r_{F}$

$D_{K S}$

$W^{2}$

$A^{2}$

$T$

$r_{10}^{(2)}$

$u$

$D_{a}$

$\chi^{2}$ likelihood ratio test for normal mixture

Engelman-Hartigan test

skewness test

kurtosis test

combined skewness-kurtosis test

5 th moment test

6th moment test

Geary's test

3rd absolute moment test

5th absolute moment test

Wilk-Shapiro test

Shapiro-Francia test

probability plot correlation

Kolmogorov-Smirnov test

Cramer-von Mises test

Anderson-Darling test

Grubbs' outlier test

Dixon's outlier test

range test

D'Agostino's D

$\chi^{2}$ test 
Table 2. Power of Tests for $n=25$ at .05 Level of Significance (\%).

Distance between Means

Test

1

2

3

4

5

Mixing Proportion $\pi=.50$

\begin{tabular}{|c|c|c|c|c|c|}
\hline LRT & $x$ & 8.5 & 31.1 & 76.3 & 98.1 \\
\hline EH & $x$ & 12.6 & 42.9 & 85.2 & 98.9 \\
\hline$\sqrt{b_{1}}$ & $x$ & 2.0 & 1.2 & 1.3 & 1.5 \\
\hline$b_{2}$ & $x$ & 7.8 & 29.6 & 62.7 & 84.5 \\
\hline$K^{2}$ & $x$ & 2.1 & 6.2 & 26.7 & 61.1 \\
\hline$m(5)$ & $x$ & 1.5 & 0.4 & 0.2 & 0.2 \\
\hline$m(6)$ & $x$ & 7.4 & 26.4 & 55.7 & 78.8 \\
\hline$a(1)$ & $x$ & 8.9 & 32.0 & 71.1 & 89.5 \\
\hline$a(3)$ & $x$ & 7.9 & 31.4 & 65.8 & 86.8 \\
\hline$a(5)$ & $x$ & 7.3 & 27.1 & 58.4 & 81.5 \\
\hline$w$ & $x$ & 5.7 & 19.0 & 55.5 & 89.5 \\
\hline $\boldsymbol{r}_{\boldsymbol{F}}$ & $x$ & 2.4 & 6.5 & 27.8 & 67.5 \\
\hline$D_{K S}$ & $x$ & 5.2 & 15.6 & 44.2 & 78.2 \\
\hline$W^{2}$ & $\mathbf{x}$ & 5.9 & 19.5 & 59.8 & 92.9 \\
\hline$A^{2}$ & $x$ & 5.8 & 19.2 & 58.8 & 92.5 \\
\hline$T$ & $x$ & 15.2 & 11.1 & 5.9 & 2.2 \\
\hline$r_{10}^{(2)}$ & $x$ & 5.1 & 6.4 & 7.8 & 10.6 \\
\hline$u$ & $x$ & 7.7 & 25.4 & 51.2 & 80.2 \\
\hline$D_{a}$ & $x$ & 4.9 & 7.9 & 3.3 & 1.1 \\
\hline$\chi^{2}$ & $x$ & 6.9 & 14.6 & 38.9 & 71.4 \\
\hline
\end{tabular}

Mixing Proportion $\pi=.60$

\begin{tabular}{|c|c|c|c|c|c|}
\hline LRT & $x$ & 8.6 & 32.5 & 78.5 & 97.6 \\
\hline EH & $x$ & 10.9 & 38.3 & 81.4 & 97.5 \\
\hline$\sqrt{b_{1}}$ & $x$ & 3.0 & 3.5 & 5.1 & 7.2 \\
\hline$b_{2}$ & $x$ & 7.1 & 23.2 & 51.0 & 68.3 \\
\hline$K^{2}$ & $x$ & 2.7 & 5.3 & 23.1 & 47.6 \\
\hline$m(5)$ & $x$ & 2.1 & 1.2 & 1.0 & 0.8 \\
\hline$m(6)$ & $x$ & 6.7 & 20.3 & 45.7 & 62.7 \\
\hline$a(1)$ & $x$ & 7.3 & 25.6 & 57.2 & 75.2 \\
\hline$a(3)$ & $x$ & 7.1 & 24.2 & 53.8 & 71.0 \\
\hline$a(5)$ & $x$ & 6.6 & 21.4 & 48.2 & 65.2 \\
\hline$W$ & $x$ & 6.9 & 21.9 & 59.9 & 90.8 \\
\hline $\boldsymbol{r}_{\boldsymbol{F}}$ & $\mathbf{x}$ & 3.2 & 9.6 & 35.0 & 73.0 \\
\hline$D_{\boldsymbol{K} S}$ & $x$ & 6.1 & 17.9 & 49.5 & 81.2 \\
\hline$W^{2}$ & $x$ & 6.3 & 23.8 & 64.8 & 93.1 \\
\hline$A^{2}$ & $x$ & 6.1 & 23.3 & 63.8 & 92.8 \\
\hline$T$ & $x$ & 12.3 & 9.7 & 4.9 & 2.2 \\
\hline$r_{10}^{(2)}$ & $x$ & 5.6 & 6.7 & 8.1 & 11.1 \\
\hline$u$ & $x$ & 7.7 & 21.5 & 48.5 & 72.3 \\
\hline$D_{a}$ & $x$ & 4.0 & 6.1 & 3.8 & 3.8 \\
\hline$\chi^{2}$ & $x$ & 7.4 & 16.7 & 44.1 & 75.0 \\
\hline
\end{tabular}


Table 2 (cont'd). Power of Tests for $n=25$ at .05 Level of Significance (\%).

Distance between Means

$\begin{array}{llllll}\text { Test } & 1 & 2 & 3 & 4 & 5\end{array}$

\begin{tabular}{|c|c|c|c|c|c|}
\hline \multicolumn{6}{|c|}{ Mixing Proportion $\pi=.65$} \\
\hline LRT & $x$ & 8.5 & 32.3 & 78.0 & 97.9 \\
\hline EH & $x$ & 9.5 & 34.5 & 74.8 & 94.9 \\
\hline$\sqrt{b_{1}}$ & $x$ & 4.2 & 6.5 & 11.2 & 17.1 \\
\hline$b_{2}$ & $\mathbf{x}$ & 6.3 & 18.3 & 36.7 & 52.1 \\
\hline$K^{2}$ & $x$ & 3.2 & 5.8 & 17.4 & 38.5 \\
\hline$m(5)$ & $x$ & 2.9 & 2.6 & 2.6 & 3.3 \\
\hline$m(6)$ & $x$ & 6.1 & 16.3 & 32.6 & 48.0 \\
\hline$a(1)$ & $x$ & 6.5 & 19.5 & 41.8 & 59.3 \\
\hline$a(3)$ & $x$ & 6.3 & 19.2 & 38.5 & 54.5 \\
\hline$a(5)$ & $x$ & 6.2 & 17.1 & 34.2 & 49.6 \\
\hline$W$ & $x$ & 7.2 & 24.3 & 63.3 & 91.7 \\
\hline$r_{F}$ & $x$ & 4.5 & 12.8 & 41.1 & 77.4 \\
\hline$D_{K S}$ & $x$ & 6.6 & 20.7 & 53.1 & 84.3 \\
\hline$W^{2}$ & $x$ & 7.4 & 25.7 & 67.8 & 94.3 \\
\hline$A^{2}$ & $x$ & 7.2 & 25.1 & 67.1 & 94.0 \\
\hline$T$ & $x$ & 12.0 & 10.2 & 6.1 & 4.1 \\
\hline$r_{10}^{(2)}$ & $x$ & 5.4 & 6.5 & 7.8 & 10.2 \\
\hline$u$ & $\mathbf{x}$ & 7.1 & 18.3 & 39.5 & 62.6 \\
\hline$D_{a}$ & $x$ & 4.0 & 5.9 & 5.2 & 10.1 \\
\hline$\chi^{2}$ & $\mathbf{x}$ & 7.8 & 17.9 & 47.8 & 76.8 \\
\hline \multicolumn{6}{|c|}{ Mixing Proportion $\pi=.70$} \\
\hline LRT & $x$ & 8.8 & 32.2 & 76.7 & 98.0 \\
\hline $\mathbf{E H}$ & $x$ & 8.7 & 26.9 & 66.0 & 91.2 \\
\hline$\sqrt{b_{1}}$ & $x$ & 5.6 & 10.6 & 21.4 & 32.6 \\
\hline$b_{2}$ & $x$ & 5.8 & 12.0 & 23.8 & 32.6 \\
\hline$K^{2}$ & $x$ & 4.2 & 7.1 & 17.3 & 32.1 \\
\hline$m(5)$ & $x$ & 4.2 & 4.6 & 7.0 & 7.5 \\
\hline$m(6)$ & $x$ & 5.8 & 10.8 & 21.2 & 29.5 \\
\hline$a(1)$ & $x$ & 5.8 & 12.8 & 27.7 & 38.6 \\
\hline$a(3)$ & $x$ & 5.4 & 12.5 & 25.4 & 34.8 \\
\hline$a(5)$ & $x$ & 5.6 & 11.2 & 22.1 & 30.9 \\
\hline$\dot{W}$ & $x$ & 8.6 & 27.3 & 67.6 & 94.1 \\
\hline$r_{F}$ & $x$ & 6.0 & 16.2 & 48.9 & 83.1 \\
\hline$D_{K S}$ & $x$ & 7.8 & 23.4 & 56.5 & 87.2 \\
\hline$W^{2}$ & $x$ & 8.1 & 28.3 & 70.0 & 95.7 \\
\hline$A^{2}$ & $x$ & 8.1 & 28.3 & 70.2 & 95.6 \\
\hline$T$ & $x$ & 11.0 & 11.4 & 9.3 & 7.0 \\
\hline$r_{10}^{(2)}$ & $\mathbf{x}$ & 5.4 & 6.0 & 7.4 & 9.7 \\
\hline$u$ & $x$ & 6.6 & 14.8 & 31.5 & 50.5 \\
\hline$D_{\mathrm{a}}$ & $x$ & 4.5 & 5.0 & 9.8 & 20.1 \\
\hline$\chi^{2}$ & $x$ & 8.5 & 18.5 & 47.1 & 79.1 \\
\hline
\end{tabular}


Table 2 (cont'd). Power of Tests for $n=25$ at .05 Level of Significance (\%).

Distance between Means

$\begin{array}{llllll}\text { Test } & 1 & 2 & 3 & 4 & 5\end{array}$

\begin{tabular}{|c|c|c|c|c|c|}
\hline \multicolumn{6}{|c|}{ Mixing Proportion $\pi=.80$} \\
\hline LRT & $\mathbf{x}$ & 8.8 & 32.6 & 72.7 & 95.5 \\
\hline EH & $\mathbf{x}$ & 5.3 & 13.9 & 40.2 & 69.8 \\
\hline$\sqrt{b_{1}}$ & $x$ & 10.4 & 26.0 & 49.9 & 70.6 \\
\hline$b_{2}$ & $x$ & 5.1 & 7.3 & 12.7 & 20.3 \\
\hline$K^{2}$ & $x$ & 8.4 & 18.3 & 34.3 & 51.1 \\
\hline$m(5)$ & $x$ & 8.5 & 16.0 & 26.0 & 34.8 \\
\hline$m(6)$ & $x$ & 4.9 & 6.9 & 11.3 & 16.9 \\
\hline$a(1)$ & $x$ & 5.0 & 8.0 & 14.7 & 25.5 \\
\hline$a(3)$ & $x$ & 4.9 & 7.3 & 13.4 & 22.5 \\
\hline$a(5)$ & $x$ & 5.0 & 7.1 & 11.8 & 18.3 \\
\hline$W$ & $x$ & 10.9 & 34.5 & 72.3 & 95.3 \\
\hline $\boldsymbol{r}_{\boldsymbol{F}}$ & $x$ & 9.2 & 28.1 & 63.9 & 91.5 \\
\hline$D_{K S}$ & $x$ & 8.3 & 26.7 & 60.2 & 86.8 \\
\hline$W^{2}$ & $x$ & 9.3 & 33.2 & 70.9 & 94.0 \\
\hline$A^{2}$ & $x$ & 9.5 & 34.1 & 72.5 & 95.3 \\
\hline$T$ & $x$ & 11.9 & 17.5 & 22.1 & 25.9 \\
\hline$r_{10}^{(2)}$ & $x$ & 6.1 & 7.5 & 8.3 & 10.4 \\
\hline$u$ & $x$ & 4.9 & 7.7 & 13.9 & 24.4 \\
\hline$D_{a}$ & $x$ & 5.1 & 10.8 & 27.1 & 52.1 \\
\hline$x^{2}$ & $\mathbf{x}$ & 8.4 & 19.3 & 46.1 & 74.6 \\
\hline \multicolumn{6}{|c|}{ Mixing Proportion $\pi=.85$} \\
\hline LRT & $\mathbf{x}$ & 8.9 & 28.7 & 67.8 & 91.4 \\
\hline EH & $\mathbf{x}$ & 4.1 & 7.4 & 25.7 & 50.6 \\
\hline$\sqrt{b_{1}}$ & $\mathbf{x}$ & 12.0 & 31.3 & 62.0 & 83.5 \\
\hline$b_{2}$ & $\mathbf{x}$ & 5.2 & 9.9 & 19.4 & 31.5 \\
\hline$K^{2}$ & $x$ & 10.5 & 23.8 & 47.7 & 69.3 \\
\hline$m(5)$ & $\mathbf{x}$ & 10.8 & 22.2 & 40.6 & 56.0 \\
\hline$m(6)$ & $\mathbf{x}$ & 5.4 & 9.5 & 17.4 & 26.6 \\
\hline$a(1)$ & $x$ & 5.1 & 8.5 & 20.0 & 35.1 \\
\hline$a(3)$ & $x$ & 4.8 & 9.7 & 19.8 & 33.6 \\
\hline$a(5)$ & $\mathbf{x}$ & 5.2 & 9.7 & 18.2 & 28.6 \\
\hline$W$ & $x$ & 11.7 & 33.6 & 71.7 & 92.6 \\
\hline$r_{F}$ & $x$ & 11.1 & 31.0 & 67.3 & 91.8 \\
\hline$D_{K S}$ & $x$ & 8.6 & 23.5 & 54.8 & 81.4 \\
\hline$W^{2}$ & $x$ & 9.6 & 28.8 & 65.5 & 88.8 \\
\hline$A^{2}$ & $x$ & 10.2 & 31.1 & 69.6 & 91.0 \\
\hline$T$ & $x$ & 12.0 & 21.4 & 3ค.7 & 42.5 \\
\hline$r_{10}^{(2)}$ & $x$ & 6.5 & 8.1 & 10.8 & 12.8 \\
\hline$u$ & $x$ & 5.3 & 6.6 & 9.6 & 15.2 \\
\hline$D_{a}$ & $x$ & 5.9 & 14.3 & 37.1 & 67.6 \\
\hline$x^{2}$ & $x$ & 8.6 & 16.6 & 39.7 & 65.3 \\
\hline
\end{tabular}


Table 2 (cont'd). Power of Tests for $n=25$ at .05 Level of Significance (\%).

Distance between Means

Test

1

2

3

4

5

Mixing Proportion $\pi=.90$

\begin{tabular}{|c|c|c|c|c|c|}
\hline LRT & $x$ & 7.5 & 25.1 & 58.2 & 81.7 \\
\hline EH & $x$ & 3.4 & 4.2 & 11.3 & 28.7 \\
\hline$\sqrt{b_{1}}$ & $x$ & 11.2 & 34.2 & 65.4 & 84.6 \\
\hline$b_{2}$ & $x$ & 6.0 & $1 \measuredangle .2$ & 30.9 & 48.4 \\
\hline$K^{2}$ & $x$ & 10.5 & 29.8 & 59.2 & 79.5 \\
\hline$m(5)$ & $x$ & 11.7 & 29.1 & 55.1 & 73.0 \\
\hline$m(6)$ & $x$ & 6.3 & 13.9 & 28.4 & 43.4 \\
\hline$a(1)$ & $\mathbf{x}$ & 5.4 & 12.6 & 28.7 & 48.3 \\
\hline$a(3)$ & $x$ & 5.4 & 13.7 & 30.9 & 50.3 \\
\hline$a(5)$ & $x$ & 6.1 & 14.1 & 30.0 & 45.9 \\
\hline$W$ & $x$ & 10.2 & 31.0 & 64.5 & 84.8 \\
\hline$r_{F}$ & $x$ & 10.0 & 31.9 & 64.8 & 85.8 \\
\hline$D_{K S}$ & $x$ & 7.3 & 20.3 & 44.4 & 67.4 \\
\hline$W^{2}$ & $x$ & 8.0 & 25.2 & 54.0 & 76.3 \\
\hline$A^{2}$ & $x$ & 8.5 & 27.6 & 58.9 & 80.8 \\
\hline$T$ & $x$ & 10.8 & 25.5 & 43.6 & 57.8 \\
\hline$r_{10}^{(2)}$ & $x$ & 6.8 & 10.6 & 16.3 & 21.9 \\
\hline$u$ & $x$ & 5.7 & 8.0 & 11.6 & 16.9 \\
\hline$D_{a}$ & $x$ & 6.0 & 17.6 & 45.4 & 73.1 \\
\hline$\chi^{2}$ & $\mathbf{x}$ & 8.6 & 15.2 & 31.0 & 51.8 \\
\hline
\end{tabular}

Mixing Proportion $\pi=.95$

\begin{tabular}{|c|c|c|c|c|c|}
\hline LRT & $x$ & 6.1 & 16.7 & 39.2 & 60.3 \\
\hline EH & $x$ & 3.0 & 2.9 & 4.2 & 10.6 \\
\hline$\sqrt{b_{1}}$ & $x$ & 10.4 & 26.7 & 50.6 & 67.6 \\
\hline $\boldsymbol{b}_{\mathbf{2}}$ & $x$ & 7.0 & 15.9 & 34.6 & 54.4 \\
\hline$K^{2}$ & $x$ & 10.2 & 26.1 & 51.0 & 67.5 \\
\hline$m(5)$ & $x$ & 11.4 & 27.3 & 50.7 & 67.0 \\
\hline$m(6)$ & $x$ & 7.1 & 15.7 & 33.9 & 52.2 \\
\hline$a(1)$ & $x$ & 6.2 & 12.0 & 27.6 & 46.9 \\
\hline$a(3)$ & $x$ & 6.4 & 15.0 & 33.5 & 54.5 \\
\hline$a(5)$ & $x$ & 7.1 & 16.0 & 34.4 & 53.3 \\
\hline$W$ & $x$ & 8.7 & 22.9 & 46.4 & 64.9 \\
\hline $\boldsymbol{r}_{\boldsymbol{F}}$ & $x$ & 9.3 & 24.7 & 48.9 & 66.9 \\
\hline$D_{K S}$ & $x$ & 6.3 & 13.5 & 26.7 & 44.0 \\
\hline$W^{2}$ & $\mathbf{x}$ & 6.8 & 16.1 & 33.7 & 52.7 \\
\hline$A^{2}$ & $x$ & 7.4 & 18.2 & 39.4 & 58.3 \\
\hline$T$ & $x$ & 10.2 & 23.5 & 43.3 & 59.3 \\
\hline$r_{10}^{(2)}$ & $\mathbf{x}$ & 7.5 & 13.2 & 21.3 & 31.8 \\
\hline $\boldsymbol{u}$ & $x$ & 6.4 & 9.8 & 15.9 & 24.6 \\
\hline$D_{a}$ & $\mathbf{x}$ & 6.4 & 15.9 & 38.3 & 59.7 \\
\hline$\chi^{2}$ & $x$ & 7.9 & 11.1 & 19.5 & 32.9 \\
\hline
\end{tabular}


Table 3. Power of Tests for $n=50$ at .05 Level of Significance (\%).

Distance between Means

$\begin{array}{llllll}\text { Test } & 1 & 2 & 3 & 4 & 5^{*}\end{array}$

\begin{tabular}{|c|c|c|c|c|c|}
\hline \multicolumn{6}{|c|}{ Mixing Proportion $\pi=.50$} \\
\hline LRT & 4.1 & 11.9 & 58.8 & 98.0 & 100.0 \\
\hline EH & 5.9 & 22.4 & 75.9 & 99.7 & 100.0 \\
\hline$\sqrt{b_{1}}$ & 4.1 & 1.5 & 0.5 & 0.8 & 1.1 \\
\hline$b_{2}$ & 5.1 & 14.6 & 58.3 & 93.5 & 99.1 \\
\hline$K^{2}$ & 4.4 & 4.5 & 33.1 & 84.3 & 99.2 \\
\hline$m(5)$ & 4.0 & 0.9 & 0.1 & 0.0 & 0.0 \\
\hline$m(6)$ & 5.1 & 14.0 & 52.9 & 88.1 & 98.1 \\
\hline$a(1)$ & 4.6 & 14.4 & 63.3 & 96.8 & 99.4 \\
\hline$a(3)$ & 4.8 & 14.9 & 61.3 & 95.5 & 99.3 \\
\hline$a(5)$ & 5.1 & 14.3 & 55.1 & 90.9 & 98.4 \\
\hline$W$ & 4.0 & 8.8 & 43.9 & 92.4 & 100.0 \\
\hline $\boldsymbol{r}_{\boldsymbol{F}}$ & 3.7 & 2.7 & 17.3 & 74.1 & 99.2 \\
\hline$D_{K S}$ & 4.6 & 6.6 & 31.9 & 83.1 & 99.0 \\
\hline$W^{2}$ & 4.3 & 8.2 & 43.4 & 94.1 & 100.0 \\
\hline$A^{2}$ & 4.4 & 7.8 & 43.4 & 93.8 & 100.0 \\
\hline$T$ & 7.4 & 8.6 & 3.9 & 1.1 & 0.0 \\
\hline$r_{10}^{(\overline{2})}$ & 4.7 & 4.8 & 5.0 & 6.7 & 8.4 \\
\hline$u$ & 4.6 & 10.3 & 36.0 & 73.5 & 95.7 \\
\hline$D_{a}$ & 4.7 & 10.4 & 24.5 & 10.1 & 0.8 \\
\hline$\chi^{2}$ & 5.6 & 7.6 & 25.6 & 73.3 & 98.1 \\
\hline \multicolumn{6}{|c|}{ Mixing Proportion $\pi=.60$} \\
\hline LRT & 4.4 & 12.5 & 62.5 & 98.2 & 100.0 \\
\hline EH & 6.6 & 19.7 & 71.9 & 98.9 & 100.0 \\
\hline$\sqrt{b_{1}}$ & 4.0 & 3.0 & 4.4 & 6.7 & 9.6 \\
\hline$b_{2}$ & 5.5 & 12.2 & 45.8 & 79.3 & 90.1 \\
\hline$K^{2}$ & 4.2 & 5.0 & 26.5 & 74.0 & 95.0 \\
\hline$m(5)$ & 3.9 & 1.4 & 0.4 & 0.1 & 0.1 \\
\hline$m(6)$ & 5.3 & 12.2 & 40.8 & 74.2 & 88.4 \\
\hline$a(1)$ & 5.6 & 11.5 & 51.0 & 84.5 & 93.3 \\
\hline$a(3)$ & 5.5 & 12.0 & 48.5 & 82.2 & 91.7 \\
\hline$a(5)$ & 5.3 & 12.2 & 43.0 & 76.5 & 89.2 \\
\hline$W$ & 4.3 & 10.0 & 48.9 & 93.9 & 100.0 \\
\hline $\boldsymbol{r}_{\boldsymbol{F}}$ & 4.0 & 4.0 & 24.0 & 78.1 & 99.2 \\
\hline$D_{K S}$ & 4.7 & 7.9 & 37.9 & 86.5 & 99.5 \\
\hline$W^{2}$ & 4.2 & 9.6 & 50.8 & 95.6 & 100.0 \\
\hline$A^{2}$ & 4.3 & 9.5 & 50.7 & 95.3 & 100.0 \\
\hline$T$ & 6.7 & 7.2 & 2.9 & 1.1 & 0.2 \\
\hline$r_{10}^{(2)}$ & 4.6 & 4.3 & 5.3 & 6.5 & 9.3 \\
\hline$u$ & 5.2 & 9.0 & 30.9 & 68.0 & 90.4 \\
\hline$D_{a}$ & 4.9 & 8.8 & 15.6 & 6.1 & 2.6 \\
\hline$x^{2}$ & 5.6 & 8.2 & 30.0 & 78.3 & 98.4 \\
\hline
\end{tabular}

*Simulation results based on 1000 samples 
Table 3 (cont'd). Power of Tests for $n=50$ at .05 Level of Significance (\%).

Distance between Means

\begin{tabular}{|c|c|c|c|c|c|}
\hline Test & 1 & 2 & 3 & 4 & $5^{*}$ \\
\hline \multicolumn{6}{|c|}{ Mixing Proportion $\pi=.65$} \\
\hline LRT & 5.0 & 13.7 & 62.6 & 98.3 & 100.0 \\
\hline EH & 6.5 & 16.0 & 64.5 & 97.4 & 100.0 \\
\hline$\sqrt{b_{1}}$ & 4.4 & 5.4 & 10.1 & 18.9 & 26.7 \\
\hline$b_{2}$ & 5.7 & 8.9 & 32.7 & 60.4 & 76.0 \\
\hline$K^{2}$ & 4.5 & 4.6 & 20.3 & 62.2 & 93.4 \\
\hline$m(5)$ & 4.4 & 2.5 & 1.0 & 0.9 & 0.6 \\
\hline$m(6)$ & 5.2 & 8.8 & 29.6 & 55.8 & 72.7 \\
\hline$a(1)$ & 5.3 & 8.1 & 35.2 & 64.4 & 78.8 \\
\hline$a(3)$ & 5.6 & 8.6 & 34.1 & 62.3 & 77.6 \\
\hline$a(5)$ & 5.4 & 8.9 & 30.8 & 57.8 & 74.6 \\
\hline$W$ & 4.1 & 11.0 & 51.8 & 94.7 & 100.0 \\
\hline $\boldsymbol{r}_{\boldsymbol{F}}$ & 4.2 & 6.1 & 29.2 & 83.6 & 99.6 \\
\hline$D_{K \boldsymbol{S}}$ & 5.3 & 9.8 & 41.7 & 87.9 & 99.6 \\
\hline$W^{2}$ & 5.0 & 11.1 & 54.4 & 96.4 & 100.0 \\
\hline$A^{2}$ & 4.9 & 11.2 & 54.3 & 96.3 & 100.0 \\
\hline$T$ & 6.3 & 7.7 & 4.0 & 1.6 & 0.2 \\
\hline$r_{10}^{(2)}$ & 5.2 & 4.8 & 4.7 & 7.7 & 8.0 \\
\hline$u$ & 4.5 & 7.2 & 25.7 & 60.1 & 86.1 \\
\hline$D_{a}$ & 4.3 & 6.6 & 9.3 & 6.1 & 11.4 \\
\hline$x^{2}$ & 6.0 & 8.3 & 33.2 & 80.3 & 98.6 \\
\hline
\end{tabular}

Mixing Proportion $\pi=.70$

$\begin{array}{rrrrrr}\text { LRT } & 4.8 & 14.0 & 62.8 & 98.4 & 100.0 \\ \text { EH } & 6.1 & 13.6 & 52.2 & 92.5 & 99.2 \\ \sqrt{b_{1}} & 5.0 & 8.3 & 21.0 & 40.0 & 56.0 \\ b_{2} & 4.7 & 7.4 & 18.3 & 36.1 & 46.4 \\ K^{2} & 4.6 & 5.6 & 17.2 & 52.9 & 89.3 \\ m(5) & 4.0 & 3.8 & 3.8 & 3.8 & 5.0 \\ m(6) & 4.5 & 7.6 & 16.9 & 33.1 & 42.8 \\ a(1) & 4.8 & 6.7 & 18.7 & 39.6 & 51.7 \\ a(3) & 4.7 & 7.3 & 18.9 & 38.1 & 48.3 \\ a(5) & 4.6 & 7.6 & 17.6 & 34.6 & 44.5 \\ W & 4.4 & 12.3 & 55.0 & 96.1 & 100.0 \\ r_{F} & 4.0 & 8.2 & 37.7 & 88.9 & 99.7 \\ D_{K S} & 5.0 & 11.3 & 46.3 & 91.2 & 99.9 \\ W^{2} & 4.8 & 13.2 & 57.7 & 97.4 & 100.0 \\ A^{2} & 4.8 & 13.2 & 58.8 & 97.4 & 100.0 \\ T & 5.8 & 7.4 & 5.5 & 3.0 & 1.9 \\ r_{10}^{(2)} & 4.5 & 4.7 & 5.3 & 6.0 & 9.0 \\ u & 4.5 & 6.7 & 19.9 & 47.5 & 71.1 \\ D_{a} & 4.3 & 5.4 & 6.4 & 11.9 & 33.0 \\ \chi^{2} & 5.9 & 95 & 33.9 & 83.3 & 99.1\end{array}$

*Simulations based on 1000 samples 
Table 3 (cont'd). Power of Tests for $n=50$ at .05 Level of Significance (\%).

Distance between Means

$\begin{array}{llllll}\text { Test } & 1 & 2 & 3 & 4 & 5^{*}\end{array}$

Mixing Proportion $\pi=.80$

$\begin{array}{rrrrrr}\text { LRT } & 5.5 & 14.3 & 63.3 & 97.8 & 100.0 \\ \text { EH } & 5.7 & 6.0 & 20.8 & 64.5 & 92.6 \\ \sqrt{b_{1}} & 4.9 & 15.9 & 50.5 & 84.6 & 95.7 \\ b_{2} & 5.6 & 5.3 & 6.4 & 12.3 & 17.9 \\ K^{2} & 4.6 & 10.7 & 31.3 & 67.5 & 93.4 \\ m(5) & 4.4 & 8.8 & 18.3 & 31.6 & 41.9 \\ m(6) & 5.2 & 4.9 & 4.9 & 8.1 & 10.7 \\ a(1) & 5.5 & 5.0 & 7.6 & 17.9 & 30.0 \\ a(3) & 5.6 & 5.4 & 7.3 & 15.8 & 23.8 \\ a(5) & 5.4 & 4.9 & 5.6 & 9.6 & 13.1 \\ W & 4.8 & 13.8 & 58.9 & 96.6 & 100.0 \\ r_{F} & 5.1 & 12.8 & 54.2 & 94.9 & 100.0 \\ D_{K S} & 5.5 & 12.5 & 51.7 & 91.8 & 99.7 \\ W^{2} & 5.5 & 14.9 & 63.1 & 97.0 & 99.8 \\ A^{2} & 5.7 & 15.4 & 65.4 & 97.7 & 99.9 \\ T & 5.4 & 8.4 & 12.7 & 13.0 & 11.4 \\ r_{10}^{(2)} & 5.0 & 4.7 & 4.7 & 5.2 & 5.7 \\ u & 5.4 & 4.1 & 6.5 & 16.6 & 33.4 \\ D_{a} & 4.9 & 5.7 & 17.1 & 50.0 & 81.9 \\ \chi^{2} & 6.0 & 8.7 & 33.2 & 79.1 & 98.5\end{array}$

Mixing Proportion $\pi=.85$

$\begin{array}{rrrrrr}\text { LRT } & 4.9 & 13.9 & 58.8 & 95.7 & 99.6 \\ \text { EH } & 5.7 & 4.4 & 9.6 & 38.7 & 72.3 \\ \sqrt{b_{1}} & 4.8 & 18.4 & 60.5 & 93.0 & 99.0 \\ b_{2} & 5.4 & 7.4 & 13.7 & 26.6 & 39.7 \\ K^{2} & 5.0 & 14.4 & 44.8 & 82.6 & 97.9 \\ m(5) & 4.8 & 12.7 & 32.7 & 58.0 & 73.1 \\ m(6) & 5.2 & 6.1 & 9.9 & 17.3 & 25.7 \\ a(1) & 5.1 & 6.3 & 13.5 & 34.4 & 54.0 \\ a(3) & 5.6 & 7.6 & 15.5 & 32.1 & 49.3 \\ a(5) & 5.3 & 6.6 & 11.2 & 20.8 & 31.9 \\ W & 4.1 & 14.2 & 56.8 & 94.9 & 99.6 \\ r_{F} & 4.6 & 16.2 & 58.7 & 95.4 & 99.8 \\ D_{K S} & 5.2 & 12.3 & 46.9 & 87.6 & 98.1 \\ W^{2} & 5.1 & 14.3 & 57.0 & 94.2 & 99.3 \\ A^{2} & 4.9 & 15.7 & \mathbf{6 0 . 3} & 95.7 & 99.5 \\ T & 5.4 & 10.6 & 19.2 & 25.9 & 29.4 \\ r_{10}^{(2)} & 4.5 & 5.2 & 5.4 & 5.6 & 7.0 \\ u & 5.0 & 4.3 & 5.2 & 7.7 & 15.0 \\ D_{a} & 4.9 & 8.2 & 28.6 & 70.2 & 94.0 \\ \chi^{2} & 5.5 & 8.6 & 28.9 & 69.8 & 92.0\end{array}$


Table 3 (cont'd). Power of Tests for $n=50$ at .05 Level of Significance (\%).

Distance between Means

$\begin{array}{llllll}\text { Test } & 1 & 2 & 3 & 4 & 5 *\end{array}$

Mixing Proportion $\pi=.90$

$\begin{array}{rrrrrr}\text { LRT } & 5.2 & 11.6 & 49.9 & 89.2 & 98.5 \\ \text { EH } & 6.1 & 3.2 & 3.1 & 13.3 & 41.1 \\ \sqrt{b_{1}} & 5.9 & 17.5 & 59.9 & 92.8 & 98.8 \\ b_{2} & 5.4 & 8.9 & 24.3 & 53.3 & 71.9 \\ K^{2} & 5.7 & 15.3 & 52.5 & 89.6 & 98.7 \\ m(5) & 5.4 & 14.1 & 44.9 & 79.9 & 92.9 \\ m(6) & 5.1 & 7.7 & 19.2 & 39.6 & 56.9 \\ a(1) & 6.0 & 7.1 & 20.4 & 53.9 & 79.0 \\ a(3) & 5.4 & 9.2 & 26.6 & 59.4 & 78.6 \\ a(5) & 5.2 & 8.1 & 21.1 & 45.2 & 63.0 \\ W & 4.9 & 12.2 & 50.0 & 88.1 & 97.8 \\ r_{F} & 5.7 & 15.5 & 56.3 & 92.2 & 98.9 \\ D_{K S} & 5.7 & 10.5 & 36.4 & 74.3 & 92.4 \\ W^{2} & 5.9 & 11.8 & 45.1 & 83.0 & 95.2 \\ A^{2} & 5.8 & 13.1 & 50.8 & 87.0 & 96.9 \\ T & 4.8 & 11.5 & 26.7 & 44.7 & 56.1 \\ r_{10}^{(2)} & 5.1 & 5.7 & 7.4 & 7.7 & 8.4 \\ u & 5.3 & 5.6 & 7.1 & 9.6 & 12.5 \\ D_{a} & 4.9 & 9.3 & 35.8 & 80.1 & 96.8 \\ \chi^{2} & 6.8 & 8.2 & 21.0 & 50.2 & 76.9\end{array}$

Mixing Proportion $\pi=.95$

\begin{tabular}{rrrrrr} 
LRT & 4.8 & 8.2 & 33.0 & 67.7 & 86.5 \\
EH & 4.9 & 3.6 & 1.6 & 1.9 & 7.7 \\
$\sqrt{b_{1}}$ & 5.1 & 13.2 & 44.3 & 75.4 & 89.2 \\
$b_{2}$ & 5.3 & 9.4 & 31.0 & 65.4 & 87.9 \\
$K^{2}$ & 5.3 & 13.4 & 44.2 & 77.6 & 91.3 \\
$m(5)$ & 5.0 & 13.3 & 42.7 & 76.2 & 91.2 \\
$m(6)$ & 4.9 & 8.6 & 27.2 & 58.1 & 82.8 \\
$a(1)$ & 5.1 & 8.1 & 21.4 & 52.1 & 78.3 \\
$a(3)$ & 5.4 & 9.7 & 31.6 & 67.1 & 88.4 \\
$a(5)$ & 4.9 & 9.0 & 28.9 & 61.7 & 86.1 \\
$W$ & 4.3 & 8.0 & 31.9 & 66.2 & 85.2 \\
$r_{F}$ & 5.2 & 12.2 & 42.7 & 76.0 & 90.4 \\
$D_{K S}$ & 5.0 & 6.9 & 20.2 & 44.2 & 68.1 \\
$W^{2}$ & 5.3 & 7.8 & 25.6 & 54.0 & 75.0 \\
$A^{2}$ & 4.7 & 8.5 & 30.1 & 61.8 & 82.0 \\
$T$ & 5.3 & 10.5 & 30.4 & 58.4 & 80.1 \\
$r_{10}^{(2)}$ & 5.0 & 7.1 & 10.9 & 16.0 & 21.7 \\
$u$ & 4.7 & 7.1 & 13.7 & 23.4 & 33.8 \\
$D_{a}$ & 4.7 & 8.8 & 32.4 & 68.3 & 88.4 \\
$\chi^{2}$ & 5.7 & 6.6 & 12.4 & 25.2 & 46.5 \\
\hline
\end{tabular}

*Simulations based on 1000 samples 
Detecting Normal Mixtures

Table 4. Power of Tests for $n=100$ at .05 Level of Significance (\%).

Distance between Means

$\begin{array}{llllll}\text { Test } & 1 & 2 & 3 & 4^{*} & 5^{*}\end{array}$

\begin{tabular}{rrrrrr} 
& \multicolumn{5}{c}{ Mixing Proportion $\pi=.50$} \\
LRT & 4.5 & 19.1 & 91.2 & 100.0 & 100.0 \\
EH & 5.8 & 32.9 & 96.7 & 100.0 & 100.0 \\
$\sqrt{b_{1}}$ & 4.7 & 1.2 & 0.3 & 0.5 & 0.6 \\
$b_{2}$ & 5.4 & 25.8 & 90.3 & 99.7 & 100.0 \\
$K^{2}$ & 4.7 & 13.4 & 80.7 & 99.7 & 100.0 \\
$m(5)$ & 4.0 & 0.7 & 0.1 & 0.0 & 0.0 \\
$m(6)$ & 6.0 & 24.3 & 84.0 & 99.0 & 100.0 \\
$a(1)$ & 5.3 & 23.9 & 93.5 & 100.0 & 100.0 \\
$a(3)$ & 4.9 & 26.7 & 92.7 & 99.9 & 100.0 \\
$a(5)$ & 6.0 & 25.6 & 87.1 & 99.6 & 100.0 \\
$W^{\prime}$ & 6.5 & 22.1 & 86.3 & 100.0 & 100.0 \\
$r_{F}$ & 4.4 & 4.3 & 55.5 & 99.6 & 100.0 \\
$D_{K S}$ & 5.1 & 9.1 & 64.2 & 98.9 & 100.0 \\
$W^{2}$ & 4.9 & 12.7 & 81.1 & 99.9 & 100.0 \\
$A^{2}$ & 4.7 & 12.8 & 81.9 & 99.9 & 100.0 \\
$T$ & 7.2 & 5.7 & 1.5 & 0.4 & 0.0 \\
$r_{10}^{(2)}$ & 4.8 & 4.8 & 4.2 & 4.7 & 6.3 \\
$u$ & 5.5 & 15.7 & 59.9 & 93.6 & 100.0 \\
$D_{a}$ & 5.6 & 25.0 & 74.9 & 47.3 & 1.7 \\
$\chi^{2}$ & 5.8 & 8.7 & 50.3 & 97.6 & 100.0 \\
& & & &
\end{tabular}

Mixing Proportion $\pi=.60$

\begin{tabular}{rrrrrr} 
LRT & 5.0 & 21.2 & 91.6 & 100.0 & 100.0 \\
EH & 6.0 & 27.7 & 93.3 & 100.0 & 100.0 \\
$\sqrt{b_{1}}$ & 4.7 & 3.9 & 7.8 & 14.4 & 20.8 \\
$b_{2}$ & 5.5 & 20.4 & 76.5 & 96.3 & 99.6 \\
$K^{2}$ & 4.1 & 11.9 & 73.2 & 99.4 & 100.0 \\
$m(5)$ & 3.5 & 1.1 & 0.2 & 0.2 & 0.0 \\
$m(6)$ & 5.5 & 19.6 & 69.7 & 93.5 & 99.0 \\
$a(1)$ & 5.5 & 18.4 & 78.8 & 97.3 & 99.4 \\
$a(3)$ & 5.3 & 21.2 & 79.9 & 97.3 & 99.5 \\
$a(5)$ & 5.6 & 20.3 & 72.9 & 94.8 & 99.4 \\
$W^{\prime}$ & 6.5 & 23.2 & 88.3 & 100.0 & 100.0 \\
$r_{F}$ & 4.3 & 6.9 & 63.8 & 99.7 & 100.0 \\
$D_{K S}$ & 5.1 & 12.1 & 70.0 & 99.7 & 100.0 \\
$W^{2}$ & 5.0 & 15.6 & 85.0 & 100.0 & 100.0 \\
$A^{2}$ & 4.8 & 16.0 & 85.4 & 100.0 & 100.0 \\
$T$ & 5.7 & 4.3 & 1.6 & 0.5 & 0.1 \\
$r_{10}^{(2)}$ & 4.6 & 3.4 & 4.7 & 5.1 & 7.4 \\
$u$ & 4.6 & 14.0 & 54.2 & 88.9 & 98.8 \\
$D_{a}$ & 5.5 & 18.9 & 42.3 & 18.8 & 2.9 \\
$\chi^{2}$ & 5.9 & 9.9 & 57.0 & 98.5 & 100.0 \\
\hline
\end{tabular}

*Simulations based on 1000 samples 
Table \& (cont'd). Power of Tests for $n=100$ at .05 Level of Significance (\%).

\begin{tabular}{|c|c|c|c|c|c|}
\hline \multicolumn{6}{|c|}{ Distance between Means } \\
\hline Test & 1 & 2 & 3 & $4^{*}$ & $5^{*}$ \\
\hline \multicolumn{6}{|c|}{ Mixing Proportion $\pi=.65$} \\
\hline LRT & 4.4 & 22.8 & 92.0 & 100.0 & 100.0 \\
\hline EH & 5.6 & 22.2 & 87.6 & 99.9 & 100.0 \\
\hline$\sqrt{b_{1}}$ & 1.7 & 8.1 & 23.4 & 45.9 & 53.9 \\
\hline$b_{2}$ & 5.3 & 14.6 & 56.2 & 84.1 & 92.1 \\
\hline$K^{2}$ & 4.4 & 10.6 & 65.6 & 99.2 & 100.0 \\
\hline$m(5)$ & 4.4 & 2.2 & 1.0 & 0.6 & 0.4 \\
\hline$m(6)$ & 5.5 & 14.2 & 51.2 & 79.5 & 91.1 \\
\hline$a(1)$ & 5.4 & 12.1 & 57.5 & 85.7 & 93.1 \\
\hline$a(3)$ & 4.7 & 14.4 & 58.9 & 85.9 & 93.0 \\
\hline$a(5)$ & 5.5 & 14.7 & 53.8 & 82.1 & 91.9 \\
\hline$W^{\prime}$ & 6.2 & 25.1 & 88.9 & 100.0 & 100.0 \\
\hline $\boldsymbol{r}_{\boldsymbol{F}}$ & 4.4 & 10.2 & 70.4 & 98.8 & 100.0 \\
\hline$D_{K S}$ & 5.3 & 17.0 & 76.5 & 99.8 & 100.0 \\
\hline$W^{2}$ & 4.8 & 20.4 & 88.0 & 100.0 & 100.0 \\
\hline$A^{2}$ & 4.9 & 20.7 & 88.6 & 100.0 & 100.0 \\
\hline$T$ & 5.9 & 4.6 & 2.0 & 0.8 & 0.0 \\
\hline$r_{10}^{(\overline{2})}$ & 4.3 & 3.8 & 4.3 & 5.6 & 6.2 \\
\hline$u$ & 4.8 & 11.2 & 45.2 & 82.8 & 98.3 \\
\hline$D_{a}$ & 5.0 & 11.9 & 19.8 & 5.7 & 16.0 \\
\hline$\chi^{\overline{2}}$ & 6.0 & 12.6 & 62.5 & 99.5 & 100.0 \\
\hline \multicolumn{6}{|c|}{ Mixing Proportion $\pi=.70$} \\
\hline LRT & 4.9 & 24.9 & 93.1 & 100.0 & 100.0 \\
\hline EH & 5.2 & 15.9 & 74.8 & 99.7 & 100.0 \\
\hline$\sqrt{b_{1}}$ & 5.3 & 15.4 & 47.0 & 74.6 & 88.2 \\
\hline$b_{2}$ & 5.5 & 8.9 & 31.3 & 56.4 & 68.9 \\
\hline$K^{2}$ & 5.3 & 11.0 & 60.4 & 98.5 & 100.0 \\
\hline$m(5)$ & 4.9 & 4.1 & 4.4 & 4.9 & 3.5 \\
\hline$m(6)$ & 5.5 & 8.8 & 29.3 & 52.6 & 67.4 \\
\hline$a(1)$ & 5.4 & 8.2 & 29.6 & 58.8 & 72.1 \\
\hline$a(3)$ & 5.4 & 8.9 & 32.2 & 58.3 & 71.0 \\
\hline$a(5)$ & 5.6 & 8.9 & 30.4 & 54.6 & 68.2 \\
\hline$W^{\prime}$ & 5.8 & 26.8 & 91.2 & 100.0 & 100.0 \\
\hline $\boldsymbol{r}_{\boldsymbol{F}}$ & 4.9 & 14.9 & 78.7 & 100.0 & 100.0 \\
\hline$D_{K S}$ & 4.8 & 20.1 & 81.4 & 100.0 & 100.0 \\
\hline$W^{2}$ & 5.2 & 23.4 & 90.6 & 100.0 & 100.0 \\
\hline$A^{2}$ & 5.0 & 24.6 & 91.1 & 100.0 & 100.0 \\
\hline$T$ & 5.1 & 6.3 & 3.0 & 0.9 & 0.2 \\
\hline$r_{10}^{(2)}$ & 4.6 & 3.8 & 4.9 & 4.9 & 6.7 \\
\hline$u$ & 4.9 & 8.3 & 37.1 & 71.4 & 94.1 \\
\hline$D_{\mathrm{a}}$ & 5.6 & 7.0 & 8.4 & 14.8 & 45.2 \\
\hline$\chi^{2}$ & 5.4 & 14.0 & 65.9 & 99.8 & 100.0 \\
\hline
\end{tabular}

*Simulations based on 100 samples 
Table $\&$ (cont'd). Power of Tests for $n=100$ at .05 Level of Significance (\%).

Distance between Means

\begin{tabular}{|c|c|c|c|c|c|}
\hline Test & 1 & 2 & 3 & $4^{*}$ & $5^{*}$ \\
\hline & & Mixing & on $\pi=$ & & \\
\hline LRT & 4.4 & 26.7 & 92.8 & 100.0 & 100.0 \\
\hline EH & 4.1 & 5.9 & 29.9 & 84.3 & 98.4 \\
\hline$\sqrt{b_{1}}$ & 5.7 & 29.9 & $8 E .5$ & 99.5 & 100.0 \\
\hline$b_{2}$ & 4.8 & 5.2 & 6.1 & 10.7 & 14.4 \\
\hline$K^{2}$ & 5.3 & 19.0 & 71.0 & 99.6 & 100.0 \\
\hline$m(5)$ & 5.7 & 14.4 & 34.2 & 57.2 & 68.9 \\
\hline$m(6)$ & 4.9 & 5.4 & 5.0 & 6.4 & 7.2 \\
\hline$a(1)$ & 4.7 & 5.3 & 10.8 & 24.6 & 35.9 \\
\hline$a(3)$ & 4.2 & 4.6 & 6.5 & 13.7 & 21.0 \\
\hline$a(5)$ & 4.7 & 5.2 & 5.4 & 7.5 & 9.5 \\
\hline$W^{\prime}$ & 5.6 & 29.4 & 91.6 & 100.0 & 100.0 \\
\hline $\boldsymbol{r}_{\boldsymbol{F}}$ & 5.3 & 25.6 & 88.9 & 100.0 & 100.0 \\
\hline$D_{K S}$ & 5.7 & 22.2 & 84.4 & 100.0 & 100.0 \\
\hline$W^{2}$ & 5.3 & 27.6 & 91.9 & 100.0 & 100.0 \\
\hline$A^{2}$ & 5.0 & 29.6 & 93.3 & 100.0 & 100.0 \\
\hline$T$ & 5.9 & 9.0 & 9.4 & 8.3 & 5.2 \\
\hline$r_{10}^{(2)}$ & 5.2 & 4.2 & 4.4 & 4.7 & 5.3 \\
\hline$u$ & 4.6 & 4.9 & 11.2 & 31.2 & 58.1 \\
\hline$D_{\mathrm{a}}$ & 4.7 & 5.9 & 25.7 & 74.8 & 96.9 \\
\hline$\chi^{2}$ & 5.5 & 13.9 & 66.0 & 98.7 & 100.0 \\
\hline
\end{tabular}

Mixing Proportion $\pi=.85$

\begin{tabular}{rrrrrr} 
LRT & 4.7 & 25.7 & 89.4 & 100.0 & 100.0 \\
EH & 3.9 & 3.3 & 9.2 & 51.7 & 89.1 \\
$\sqrt{b_{1}}$ & 6.5 & 33.6 & 90.1 & 100.0 & 100.0 \\
$b_{2}$ & 5.0 & 7.2 & 16.3 & 32.4 & 48.7 \\
$K^{2}$ & 5.9 & 23.8 & 79.8 & 99.7 & 100.0 \\
$m(5)$ & 5.8 & 18.9 & 56.6 & 85.6 & 93.8 \\
$m(6)$ & 5.4 & 6.4 & 10.4 & 17.5 & 26.5 \\
$a(1)$ & 5.3 & 7.5 & 25.8 & 56.8 & 78.2 \\
$a(3)$ & 4.4 & 6.7 & 18.1 & 39.6 & 60.5 \\
$a(5)$ & 5.4 & 6.7 & 12.9 & 23.0 & 36.3 \\
$W^{\prime}$ & 5.7 & 26.5 & 87.4 & 100.0 & 100.0 \\
$r_{F}$ & 6.1 & 27.5 & 88.2 & 100.0 & 100.0 \\
$D_{K S}$ & 5.4 & 20.0 & 77.6 & 99.3 & 100.0 \\
$W^{2}$ & 5.2 & 25.2 & 86.4 & 100.0 & 100.0 \\
$A^{2}$ & 5.2 & 27.5 & 89.3 & 100.0 & 100.0 \\
$T$ & 5.5 & 10.5 & 16.0 & 18.9 & 19.9 \\
$r_{10}^{(2)}$ & 4.5 & 4.3 & 4.3 & 4.6 & 5.6 \\
$u$ & 4.9 & 4.3 & 4.4 & 12.2 & 24.3 \\
$D_{a}$ & 4.8 & 9.4 & 45.1 & 91.6 & 99.8 \\
$\chi^{2}$ & 5.3 & 12.4 & 54.1 & 95.4 & 99.8 \\
\hline
\end{tabular}

*Simulations based on 1000 samples 
Table 4 (cont'd). Power of Tests for $n=100$ at .05 Level of Significance (\%).

Distance between Means

\begin{tabular}{|c|c|c|c|c|c|}
\hline Test & 1 & 2 & 3 & $4^{*}$ & $5^{*}$ \\
\hline \multicolumn{6}{|c|}{ Mixing Proportion $\pi=.90$} \\
\hline LRT & 4.9 & 21.8 & 81.3 & 99.2 & 100.0 \\
\hline $\mathbf{E H}$ & 3.7 & 2.0 & 1.1 & 11.3 & 48.5 \\
\hline$\sqrt{b_{1}}$ & 5.9 & 31.5 & 86.5 & 99.5 & 100.0 \\
\hline$b_{2}$ & 5.6 & 10.6 & 37.2 & 74.9 & 93.6 \\
\hline$K^{2}$ & 5.7 & 25.6 & 81.6 & 99.7 & 100.0 \\
\hline$m(5)$ & 6.3 & 23.0 & 72.1 & 98.0 & 99.9 \\
\hline$m(6)$ & 6.0 & 9.7 & 27.5 & 58.5 & 78.3 \\
\hline$a(1)$ & 5.4 & 10.9 & 41.6 & 85.2 & 98.9 \\
\hline$a(3)$ & 4.9 & 9.7 & 39.8 & 81.2 & 96.5 \\
\hline$a(5)$ & 5.8 & 9.7 & 31.7 & 66.5 & 85.7 \\
\hline$W^{\prime}$ & 5.9 & 21.2 & 78.3 & 98.4 & 100.0 \\
\hline$r_{F}$ & 5.7 & 26.6 & 84.3 & 99.7 & 100.0 \\
\hline$D_{K S}$ & 5.2 & 16.4 & 63.5 & 94.1 & 99.6 \\
\hline$w^{2}$ & 5.2 & 19.8 & 73.7 & 98.1 & 99.9 \\
\hline$A^{2}$ & 5.4 & 22.7 & 79.7 & 98.8 & 100.0 \\
\hline$T$ & 6.0 & 13.5 & 28.4 & 46.8 & 58.9 \\
\hline$r_{10}^{(2)}$ & 5.5 & 5.1 & 4.4 & 4.2 & 4.3 \\
\hline$u$ & 5.0 & 5.1 & 4.8 & 5.4 & 7.6 \\
\hline$D_{\alpha}$ & 5.1 & 12.0 & 58.6 & 97.6 & 100.0 \\
\hline$x^{2}$ & 5.8 & 9.3 & 37.1 & 78.8 & 97.3 \\
\hline \multicolumn{6}{|c|}{ Mixing Proportion $\pi=.95$} \\
\hline LRT & 4.4 & 13.6 & 57.1 & 92.5 & 98.1 \\
\hline $\mathbf{E H}$ & 4.4 & 2.0 & 0.4 & 0.3 & 5.8 \\
\hline$\sqrt{b_{1}}$ & 5.8 & 20.6 & 67.7 & 93.4 & 98.0 \\
\hline$b_{2}$ & 5.9 & 12.6 & 47.5 & 90.3 & 98.3 \\
\hline$K^{2}$ & 5.9 & 20.2 & 68.3 & 95.3 & 98.4 \\
\hline$m(5)$ & 5.9 & 20.0 & 65.9 & 95.1 & 98.5 \\
\hline$m(6)$ & 6.0 & 12.0 & 41.7 & 85.8 & 97.5 \\
\hline$a(1)$ & 5.6 & 10.4 & 38.7 & 81.8 & 95.3 \\
\hline$a(3)$ & 5.1 & 10.8 & 46.1 & 89.5 & 98.1 \\
\hline$a(5)$ & 6.1 & 12.3 & 44.3 & 87.9 & 98.0 \\
\hline$W^{\prime}$ & 5.8 & 12.5 & 51.1 & 89.2 & 97.3 \\
\hline $\boldsymbol{r}_{\boldsymbol{F}}$ & 5.5 & 20.3 & 67.0 & 95.2 & 98.3 \\
\hline$D_{K S}$ & 5.3 & 9.9 & 33.8 & 72.2 & 88.9 \\
\hline$W^{2}$ & 5.5 & 11.3 & 42.3 & 78.6 & 93.0 \\
\hline$A^{2}$ & 5.4 & 13.3 & 50.3 & 85.6 & 95.4 \\
\hline$T$ & 5.8 & 12.7 & 37.9 & 75.3 & 90.9 \\
\hline$r_{10}^{(2)}$ & 4.8 & 6.9 & 8.5 & 7.8 & 7.3 \\
\hline$u$ & 5.3 & 7.8 & 14.9 & 25.8 & 29.3 \\
\hline$D_{E}$ & 5.2 & 11.8 & 50.5 & 90.6 & 98.1 \\
\hline$x^{2}$ & 5.7 & 7.2 & 16.9 & 44.7 & 77.0 \\
\hline
\end{tabular}

*Simulations based on 1000 samples 


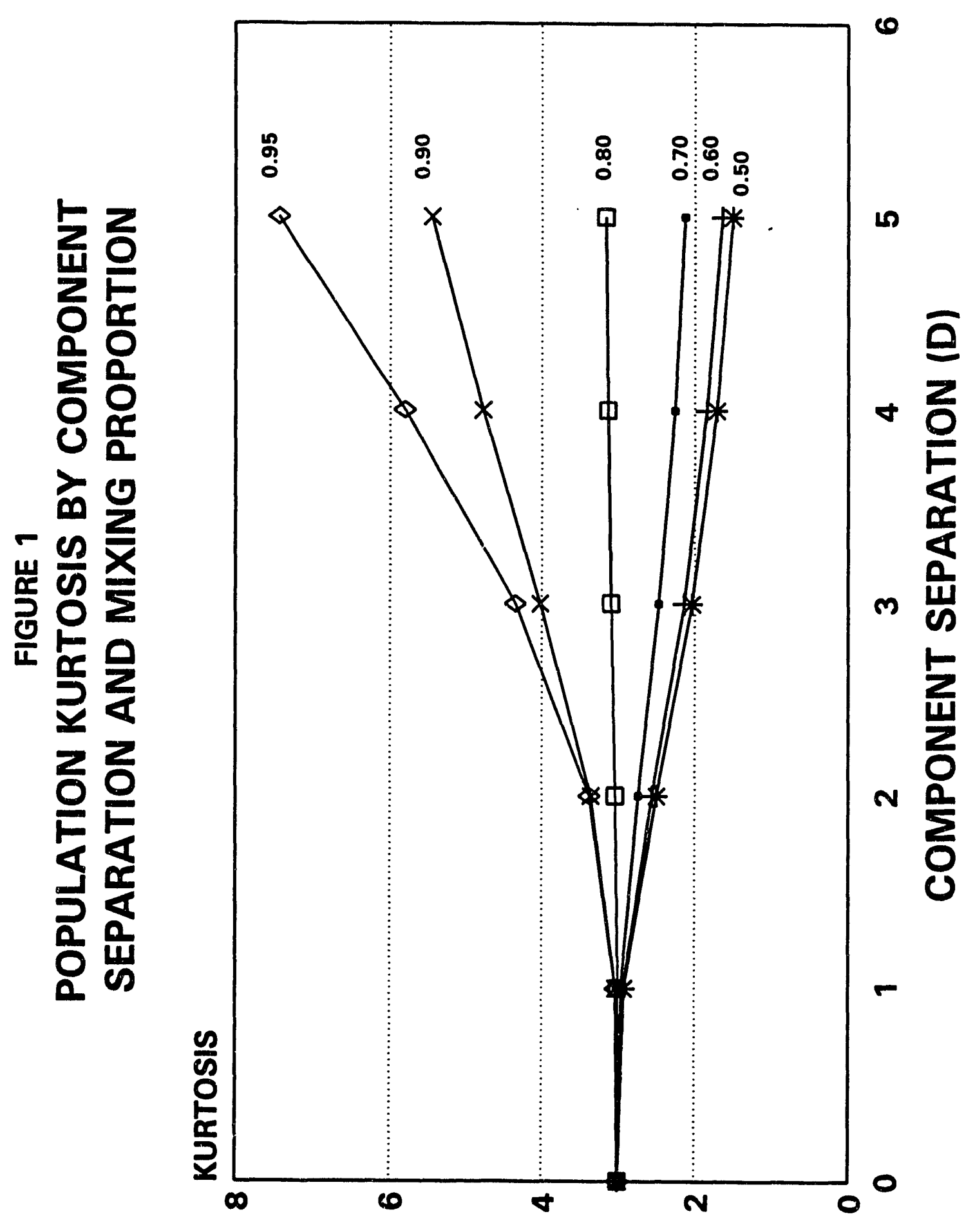




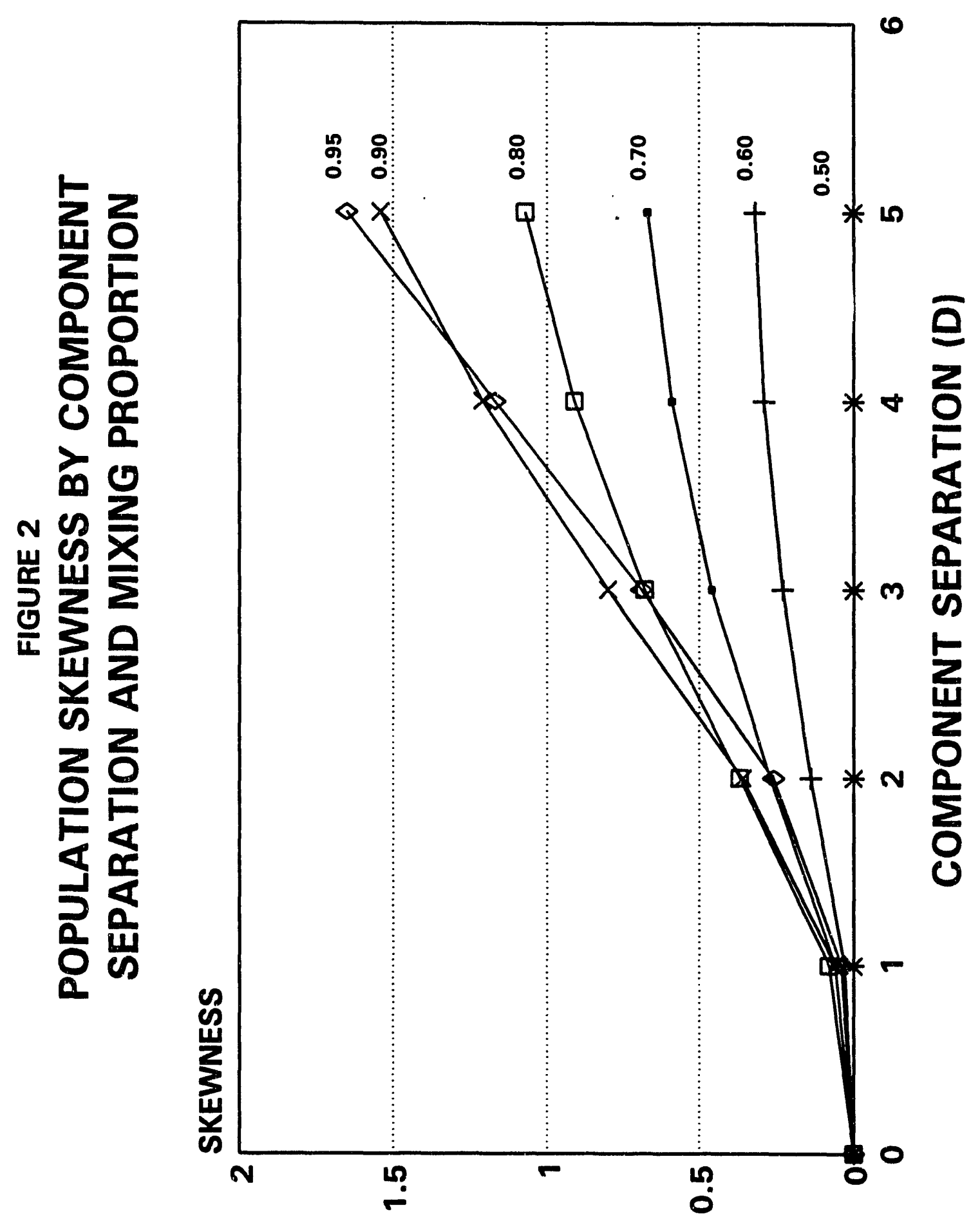


DATE

FILMED

215193 
